Conclusions The recalculation resulted in a substantial reduction in the number of measurements below the LOD. The degree of censoring after recalculation is well within the operating range of the statistical methods used in the GuLF STUDY to estimate exposure levels.

\section{THE NIEHS GULF STUDY: A COMPARISON OF THE $\beta$ - SUBSTITUTION METHOD AND A BAYESIAN APPROACH FOR HANDLING HIGHLY CENSORED MEASUREMENT DATA}

${ }^{1}$ Tran Huynh, ${ }^{2}$ Harrison Quick, 'Gurumurthy Ramachandran, ${ }^{3}$ Sudipto Baneriee, ${ }^{4}$ Joao Monteiro, ${ }^{3}$ Caroline Groth, ${ }^{5}$ Mark Stenzel, ${ }^{6}$ Aaron Blair, ${ }^{7,8}$ Dale Sandler, 7,8 Lawrence Engle, ${ }^{8}$ Richard Kwok, ${ }^{9}$ Patricia Stewart. ${ }^{1}$ Department of Environmental Health Sciences, University of Minnesota, Minneapolis, MN, USA; ${ }^{2}$ Department of Statistics, University of Missouri, Columbia, MO, USA; ${ }^{3}$ Division of Biostatistics, University of Minnesota, Minneapolis, MN, USA; ${ }^{4}$ NAMSA, Minneapolis, MN, USA; ${ }^{5}$ Exposure Assessment Applications, LLC, Arlington, VA, USA; ${ }^{6}$ National Cancer Institute, Gaithersburg, MD, USA; ${ }^{7}$ Department of Epidemiology, University of North Carolina at Chapel Hill, Chapel Hill, NC, USA; ${ }^{8}$ Epidemiology Branch, National Institute of Environmental Health Sciences, Research Triangle Park, NC, USA; ${ }^{9}$ Stewart Exposure Assessments, LLC, Arlington, VA, USA

10.1136/oemed-2014-102362.325

Objectives Over 150000 measurements taken on workers responding to the 2010 Deepwater Horizon oil spill are being used to develop exposure estimates for the participants in the GuLF STUDY. A large portion of the measurements, however, has values below the limit of detection (left-censored). The $\beta$ substitution method has been shown to provide accurate estimates for handling censored data, but a comparison to a Bayesian method, which permits the estimation of uncertainty and accounts for prior information, is currently lacking. The goal of this research was to compare the two methods.

Method Each method was challenged with computer-generated datasets drawn from lognormal distributions with the geometric mean $(\mathrm{GM})=1$, sample sizes $=5-100$, geometric standard deviation $(G S D)=2-5$, and percent censoring $=10-90 \%$. Percent bias and coverage (the percentage of $95 \%$ uncertainty intervals containing the truth) were used as evaluation metrics.

Results For most of our simulation scenarios, estimates of bias from the $\beta$-substitution and Bayesian methods were generally comparable for the AM and GM. The $\beta$-substitution was generally less biassed in estimating the GSD and the $95^{\text {th }}$ percentile than the Bayesian method. The Bayesian method provided consistently better coverage for the AM than $\beta$-substitution. It also provided uncertainty estimates the GM, GSD, and the $95^{\text {th }}$ percentile while $\beta$-substitution does not.

Conclusions The $\beta$-substitution method generally was observed to have little bias but it only allows the calculation of uncertainty estimates around the AM. The Bayesian approach provided reasonably accurate point and interval estimates (i.e., coverage), but this comes with the cost of additional computation.

\section{USE OF ACETOCHLOR AND CANCER INCIDENCE IN THE AGRICULTURAL HEALTH STUDY COHORT}

${ }^{1,2}$ Catherine Lerro, ${ }^{1}$ Stella Koutros, ${ }^{1}$ Gabriella Andreotti, ${ }^{3}$ Cynthia Hines, ${ }^{1}$ Jay Lubin, ${ }^{2}$ Yawei Zhang, 'Laura Beane Freeman. ${ }^{1}$ National Cancer Institute, Bethesda, MD, USA; ${ }^{2}$ Yale University, New Haven, CT, USA; ${ }^{3}$ National Institute for Occupational Safety and Health, Cincinnati, $\mathrm{OH}$, USA

10.1136/oemed-2014-102362.326
Objectives Since its registration in 1994, acetochlor, an herbicide licensed for use on corn, has been one of the most commonly used pesticides in the US. We evaluated use of acetochlor and cancer incidence in the Agricultural Health Study, a prospective cohort of licensed pesticide applicators.

Method During a telephone interview administered from 19992005, participants provided information on acetochlor use and other factors. Total lifetime days of acetochlor use were calculated and an intensity-weighting algorithm was applied that accounted for factors that modify exposure. We used Poisson regression to estimate relative risks (RR) and 95\% confidence intervals (CI) for cancers that occurred from the time of interview through 2011 in Iowa and 2010 in North Carolina. We examined all cancer sites together, and individual sites with $>10$ exposed cases.

Results Among 33484 men, 3234 incident cancers and 304 acetochlor-exposed cases occurred. An increased risk of lung cancer was observed among ever users of acetochlor $(n=23)$ $(\mathrm{RR}=1.57 ; \mathrm{CI}=0.95-2.59)$ compared to never users, but there was no evidence of an exposure response trend $(\mathrm{p}$-trend $=0.30)$. Also, there was increased risk of colorectal cancer $(n=25)$ with high acetochlor use $(\mathrm{RR}=1.60 ; \mathrm{CI}=0.97-2.65$, $\mathrm{p}$-trend $=0.14)$. Conclusions The associations between acetochlor use and colorectal and lung cancer are novel. However, due to lack of exposure-response trend, small number of exposed cases, and relatively short time between acetochlor use and cancer development, these findings warrant caution in interpretation and further investigation.

\section{INCIDENCE OF SICKNESS ABSENCE AMONG BRAZILIAN PUBLIC WORKERS}

${ }^{1,2}$ Ana Lucia Leao, ${ }^{2}$ Anadergh Barbosa-Branco, ${ }^{2}$ Marilia Turchi, ${ }^{1}$ Donald Cole, 1,3Ivan Steenstra. ${ }^{1}$ University of Toronto, Toronto, ON, Canada; ${ }^{2}$ Federal University of Goias, Goiania, Goias, Brazil; ${ }^{3}$ Institute for Work and Health, Toronto, ON, Canada

\subsection{6/oemed-2014-102362.327}

Objectives Investigate the incidence and risk factors of sickness absence (SA) among Brazilian public workers.

Method It is a dynamic cohort composed by all public workers of the Goiania municipality hired from January, 2005 to December, 2011 where certified SA data were analysed. Incidence density (ID) was calculated per 1.000 person-years in each ICD-10 category. We used multiple logistic regression analysis to estimate the association between SA and socioeconomic and occupational characteristics.

Results 18.450 workers contributed to 49.41006 person- years and 7.044 (38.2\%) employees had at least one certified episode of SA. The ID of sickness absence was 142.5 per 1.000 personyears. This rate was significantly higher among women (155.5). Diagnostic groups with the highest sick leave ID were for injury (32.3) and musculoskeletal diseases (15.3). The logistic analysis showed association between SA and the female gender (OR 1.46 CI95\% 1.35-1.58), low education (OR 1.34 CI95\% 1.18-1.52), low salary (OR 1.82 CI95\% 1.61-2.05), multiple work contracts (OR $1.56 \mathrm{CI} 95 \% 1.46-1.66)$ and was greater among professionals in the health field (OR 1.73 CI95\% 1.551.92).

Conclusions On average, 14\% of workers received a SA certificate each year, and the socioeconomic and occupational characteristics were associated with higher rate of certification. This study suggests an increased risk according to the professional category, the field of education was more associated with mental 
disorders, blue collar with musculoskeletal disorders and inspection with injuries. The findings provide relevant information for disease's prevention and health promotion policies with priority to the most vulnerable occupational groups.

\section{CONDITIONS OF RETURN TO WORK OF NURSES AFTER MATERNITY LEAVE}

Eduardo Sá, Renata Razaboni, Marcela Braga, Flávio Gosling, Marcelo Pustiglione. Faculdade de Medicina Da Universidade de São Paulo, São Paulo, Brazil

\subsection{6/oemed-2014-102362.328}

Objectives Currently, countries like France, Spain, Italy, United States and Brazil there was an increase in the proportion of workers aged between 25-45 years, a period considered fertile. This scenario has determined that many workers had to choose between career and motherhood. The aim is to analyse the amount of Brazilian nursing professionals, in the period from January, 2012 to July 2013, returned to work after maternity leave.

Method Was performed a literature review at Pub Med and BIREME. There was a review of the medical records of nursing professionals met at a public university hospital of São PAulo, Brazil, from January 2012 to July 2013.

Results Returned to work after maternity leave: $80 \%$ (57) of nursing assistants, 70\% (16) of the nursing staff and 94.6\% (53) of nurses. Among the layoffs, $62.5 \%$ (15) occurred within the first 3 months, and only $18.75 \%$ (3) in the first six and nine months after maternity leave.

Conclusions It is possible to conclude that the nursing professional return to work after maternity leave quality requires investment in health policies that promote greater social and labour support.

\section{THE NIEHS GULF STUDY: ESTIMATE OF WORKERS' EXPOSURES THROUGH THE INHALATION ROUTE ON SEVEN RESPONSE VESSELS NEAR THE WELL-SITE DURING THE DEEPWATER HORIZON OIL SPILL}

\footnotetext{
${ }^{1}$ Gurumurthy Ramachandran, ${ }^{1}$ Tran Huynh, ${ }^{2}$ Sudipto Banerjee, ${ }^{2}$ Caroline Groth, ${ }^{3}$ Mark Stenzel, ${ }^{4}$ Aaron Blair, ${ }^{5}$ Lawrence Engel, ${ }^{5}$ Dale Sandler, ${ }^{5}$ Richard Kwok, ${ }^{6}$ Patricia Stewart. 'Department of Environmental Health Sciences, University of Minnesota, Minneapolis, MN, USA; ' Division of Biostatistics, University of Minnesota, Minneapolis, MN, USA; ${ }^{3}$ Exposure Assessment Applications, LLC, Arlington, VA, USA; ${ }^{4}$ National Cancer Institute, Gaithersburg, MD, USA; ${ }^{5}$ Epidemiology Branch, National Institute of Environmental Health Sciences, Research Triangle Park, NC, USA; ${ }^{6}$ Stewart
} Exposure Assessments, LLC, Arlington, VA, USA

\subsection{6/oemed-2014-102362.329}

Objectives After the Deepwater Horizon, response vessels were brought near the wellhead area to stop the leak, collect oil and drill the relief wells. The objective of this paper is to characterise inhalation exposure on these vessels for various exposure groups (EGs) to total hydrocarbons (THCs).

Method Approximately 100 EGs based on job title group, tasks and time period during the response effort were standardised across these vessels. Descriptive statistics were calculated from the measurements for each EG on each vessel. Since many of the data were censored (i.e., below the limit of detection), a Bayesian method was used to obtain estimates for the arithmetic mean (AM), geometric mean, and geometric standard deviation.
Results During the first time period (April 20-May 14), the range of the AMs on the vessels was 1.9-24.2 ppm THC. Exposures were lower during the second period (May 15-July 15) when dispersant was used to reduce air concentrations (0.1-14.6 ppm). After top-capping the well, a substantial reduction was observed on all seven ships (0.1-1.9 ppm). After bottom capping (>August 10), exposures generally increased on the ships (0.1$3.8 \mathrm{ppm})$, most likely due to decontamination activities. The vessel capping the well and the vessel burning waste oil and gas had significant differences from the vessels drilling the relief wells. Conclusions Differences were found by vessel and time period that likely reflected oil weathering and differences in job and vessel activities.

\section{PARENTAL OCCUPATIONAL EXPOSURE TO IONISING RADIATION AND SELECTED BIRTH DEFECTS IN THE US}

${ }^{1} \underline{H}$ Lim, ${ }^{1} \mathrm{AJ}$ Agopian, ${ }^{1} \mathrm{LW}$ Whitehead, ${ }^{2} \mathrm{CW}$ Beasley, ${ }^{3} \mathrm{PH}$ Langlois, ${ }^{1} \mathrm{RJ}$ Emery, ${ }^{1} \mathrm{DK}$ Waller, and The National Birth Defects Preventions Study. 'University of Texas Houston Health Science Center, Houston, USA; ${ }^{2}$ University of Texas School of Medicine, Houston, USA; ${ }^{3}$ Birth Defects Epidemiology and Surveillance Branch, Texas Department of State Health Services, Austin, USA

\subsection{6/oemed-2014-102362.330}

Objectives Results of previous studies of occupational exposure to ionising radiation (IR) and birth defects are inconsistent. Our objective was to assess the association between maternal and paternal occupational exposure to ionising radiation (IR) and birth defects using a larger sample than previous studies.

Method We studied 27809 case mothers and 10200 control mothers who participated in the National Birth Defects Prevention Study (NBDPS), giving birth between 1997 and 2009. Our exposure assessment was based on a textual analysis of the mother's description of her occupation, workplace and job activities (and those of the infant's father) during the three months before and the three months after the estimated date of conception. Logistic regression was used to examine crude and adjusted odds ratios for the association between possible maternal and paternal occupational exposures to IR and 45 birth defects. We assessed the possibility of confounding from pre-pregnancy diabetes and body mass index, smoking, use of supplements containing folic acid, use of alcohol, use of illicit drugs, pregnancy intention, study location and demographic variables.

Results We excluded 17 mothers with a history of cancer, and 12568 mothers who were unemployed, homemakers or students during the periconceptional period. Overall, $3 \%$ of the mothers and $2 \%$ of the fathers were exposed to IR. The remainder of our results are underway and will be presented at the meeting. Conclusions Our results will be interpreted taking account of multiple statistical comparisons and the possibility of recall bias.

\section{MONITORING MERCURY EXPOSURE AMONG ARTISANAL AND SMALL-SCALE MINERS: DEVELOPING AND EVALUATING A SURVEILLANCE PROTOCOL}

Gabriela Gracia, Linda Forst. UIC, Chicago, IL, USA

\subsection{6/oemed-2014-102362.331}

Objectives - Develop a mercury exposure surveillance protocol targeted at artisan and small-scale miners that includes taking occupational histories, ancillary tests, counselling and educating patients regarding exposure 